\title{
LA CRIMINALIZACIÓN DEL COLECTIVO INMIGRANTE EN EL DISCURSO DE LA PRENSA
}

\author{
THE CRIMIN ALIZATION OF THE IMMIGRANT COLLECTIVE IN \\ THE PRESS DISCOURSE
}

\author{
Pedro Garrido Rodríguez' \\ Universidad de Salamanca
}

\begin{abstract}
Resumen
El discurso de la prensa con respecto a las migraciones suele caracterizarse por la simplificación, la reproducción de estereotipos, la visión parcial y la ambigüedad. En el presente artículo se emplean las metodologías de análisis de contenido y análisis crítico del discurso para estudiar el discurso de la prensa en lo referente al colectivo inmigrante. Se percibe cómo, al reflejo del discurso político, a la vez que existe un enfoque proactivo con las migraciones, minoritario, existe un robusto y creciente enfoque reactivo que, haciendo uso de sutiles herramientas lingüísticas, traslada una imagen sobredimensionada, negativa y con frecuencia criminalizadora del colectivo inmigrante. Asimismo, se detallan las principales directrices y ejemplos de buenas prácticas al efecto.
\end{abstract}

Palabras clave

Discurso de la prensa. criminalización e inmigración. Imagen del colectivo inmigrante.

Racismo discursivo. Análisis crítico del discurso.

\section{Abstract}

The press discourse about migations, oftenly contains simplification, prejuices, parcial vision and ambiguity. In this article have used the metodologies of analysis of content and analysis critical of discourse in order to study the press discourse about immigrant collective. It is perceived how, as a reflection of political discourse, while there is a proactive discourse with migrations, minority, there is a robust and growing reactive approach, that, using subtle linguistic tools, transfer san oversized, negative and often criminalizing image of the immigrant collective. The main guidelines and examples of good practices for this purpose are also detailed.

Keywords

Press speech. Criminalization and immigration. Image of the immigrant collective. Discursive racism. Critical discourse analysis.

${ }^{1}$ Doctor por la Universidad de Salamanca. Docente de los Programas de Posdoctorado de Derechos Humanos DHPCBE y GDHDS de la Universidad de Salamanca. 


\section{INTRODUCCIÓN}

El discurso de la prensa en lo referente a las migraciones suele caracterizarse por la simplificación, la no consideración de la complejidad de los conceptos, el sesgo y la sobredimensión del fenómeno. Según Van Dijk, la imagen que se elabora de las personas migrantes, las minorías, los refugiados y, en conjunto, todo lo que representa la otredad, en el discurso promovido por las élites responde a una serie de rasgos. El primero de ellos, es la estrategia general de autorrepresentación positiva y presentación negativa del otro $^{2}$. La diferencia entre "nosotros" y "ellos" es nuclear y se percibe nítidamente en los temas, el léxico, las hipérboles, los eufemismos, los descargos de responsabilidad, la narración, la argumentación, las imágenes y otras propiedades del discurso. La representación de "los otros" suele enfatizar un conjunto de temas estereotipados como la inmigración irregular, las dificultades en la recepción de inmigrantes y en su integración en la sociedad de destino, actos delictivos, drogas y conductas abyectas que se asocian al conjunto inmigrante. En conjunto, se subraya todo lo que tiene que ver con la diferencia, la desviación y la amenaza, trasladando una visión que criminaliza a priori al colectivo inmigrante.

En la estrategia de autorrepresentación positiva, de manera especial en el discurso de las élites, resulta fundamental la maniobra de negar taxativamente el racismo o, al menos, diluirlo. Cuando se aborda en prensa, generalmente es referido al racismo biológico. Ese racismo tosco, zafio y ostentoso que únicamente es sostenido desde las posiciones más extremas y que es rechazado por la mayor parte de la opinión pública. Sin embargo, en muy pocas ocasiones se reproducen noticias sobre el racismo

${ }^{2}$ VAN DIJK, Teun. "Discurso de las élites y racismo institucional”. En: Bastida, L. (Coord.). Medios de comunicación e inmigración, Murcia, Caja de Ahorros del Mediterráneo-Obra Social, 2006, pp. 15-34. 
de los grupos dominantes. Hay una importante carencia de noticias autocríticas sobre el discurso reactivo y criminalizador presente en la propia prensa.

Los discursos que tratan acerca de las minorías se encuentran esencialmente en manos de las élites blancas. Las personas pertenecientes a las minorías prácticamente no tienen acceso, representatividad, ni influencia en lo que se escribe o se dice sobre ellos. Y cuando lo tienen, no se les da la credibilidad ni la entidad que les corresponde como protagonistas de lo que están contando. Las historias referidas a ellos suelen ser parciales y condicionadas por consideraciones, juicios y sesgos del endogrupo. Asimismo, en las redacciones apenas hay presencia de profesionales que pertenezcan a minorías, y mucho menos en los puestos directivos. Así las cosas, el discurso de la prensa se gesta en un medio excluyente desde el inicio.

Por otra parte, los discursos sobre las minorías que observamos en prensa rara vez se destinan a las propias minorías que describen. Generalmente las ignoran como posibles agentes receptores. Se enfatizan los aspectos más negativos y apenas se presta atención a conocer los problemas experimentados por los inmigrantes, sus vidas, su trabajo, sus condicionantes, su contribución a la economía del país. Sobre esto ahonda Van Dijk, quien afirma que la prensa europea en general no define la situación étnica en términos de los propios protagonistas étnicos ${ }^{3}$. El discurso reivindicativo, formulado y emitido por el exogrupo, por "ellos", es percibido generalmente como incómodo y desestabilizador y suele encontrarse limitado en su proceso de transmisión.

\section{ANÁLISIS DEL SUJETO EMISOR}

${ }^{3}$ VAN DIJK, Teun (2006): Discurso de las élites... Op. Cit. Pp. 15-34. 
Bañón Hernández realiza un análisis muy esclarecedor acerca de tres tipos de discurso sobre inmigración que resultan fundamentales para poder comprender su tratamiento en los medios de comunicación ${ }^{4}$. El primero, es el discurso reivindicativo, formulado por "ellos", el exogrupo. Los otros dos, muy dispares entre sí, están formulados por "nosotros", el endogrupo: se trata del discurso comprometido y el discurso discriminador. En el análisis sobre la representación periodística acerca del discurso de la reivindicación y los reivindicadores, Bañón señala mecanismos y actuaciones de los que los medios suelen valerse para distorsionarlo, restándole significación, validez, credibilidad, legitimación, profundidad o, en conjunto, fuerza movilizadora. En primer lugar, la supresión del discurso reivindicativo y de los reivindicadores. Se opta por anular o minimizar el discurso, "no dejar decir". No obstante, una vez que el discurso reivindicativo se produce, también puede obstaculizarse su representación otorgándole espacios de baja audiencia, publicando artículos en páginas interiores, interfiriendo las frecuencias radiales, evitando que el público escuche los discursos, etc. Se trata de no facilitar la transmisión del mensaje en las condiciones propicias. En muchas ocasiones se justifica la ausencia de reivindicaciones directas de personas inmigrantes por el escaso conocimiento de la lengua española, cuestión que podría solventarse sin grandes problemas mediante el empleo de traducción simultánea o subtítulos.

Un recurso muy empleado es hacer constar la existencia de la reivindicación para omitir luego su contenido, diluyendo así el discurso reivindicativo. Se informa de que hay o ha habido un acto reivindicativo, pero no se habla de sus causas, sus argumentos, sus pretensiones. De este modo, se hace ver que los inmigrantes tienen voz, con lo que se proyecta una imagen democrática de "nosotros" - la autorrepresentación positiva de

${ }^{4}$ BAÑÓN HERNÁNDEZ, A. Discurso e inmigración. Propuestas para un debate social, Murcia, Universidad de Murcia, 2002. 
la que habla Van Dijk- pero no se hace referencia a lo sustantivo del mensaje que pretenden transmitir.

Otra forma de desacreditar el discurso reivindicativo o a sus emisores, es lo que Bañón llama desplazamiento semántico-pragmático. Consiste en servirse de asociaciones semánticas y metáforas para asociar a la inmigración con ámbitos como la mendicidad, la violencia o incluso la diversión, y focalizar la atención en ellos. De este modo, el contenido reivindicativo de la queja queda difuminado y se le resta credibilidad a sus autores. Si el acto de protesta se ha visto complicado además con algún tipo de altercado violento o ha tenido un tono que se salga en algo de lo puramente reivindicativo, también suele aprovecharse para diluir en ello la reivindicación.

En ocasiones puede manipularse su autoría presentándolo como obra de asociaciones de ayuda a la inmigración, alguna ONG u otros actores sociales siempre pertenecientes al endogrupo. De esta forma se devalúa la capacidad de los reivindicadores para asociarse y realizar planteamientos de manera normalizada y se da a entender que la reivindicación se ha producido como una autocrítica que parte de "nosotros", no como una reclamación de "ellos".

Las personas inmigrantes son representadas en ocasiones por los medios como seres cuyas peticiones resultan exageradas, que han venido con unas expectativas demasiado altas con respecto a lo que se les puede ofrecer y que no están dispuestos a negociar ni a ceder.

El fin de la Guerra Fría conllevó, además de la resolución de no pocas tensiones internacionales, el término de una serie de hábitos textuales y semánticos muy consolidados dentro del ámbito mediático ${ }^{5}$ que

${ }^{5}$ Para un conocimiento más exhaustivo de la Guerra Fría, y las estrategias de propaganda creadas en torno a ella, véanse: GADDIS, J. L. La guerra fría, Barcelona, RBA, 2008; LAFEBER, W. America, Russia, and the cold war, McGraw-Hill, 1987; VEIGA, F. y DUARTE, A. La paz simulada: una bistoria de la Guerra Fría 1941-1991, Madrid, Alianza, 1997; ARON, R. Les articles de politique internationale dans le Figaro de 1947 à 1977, París, 
fueron mutando para pasar a formar parte de otros marcos de referencia como la etnicidad ${ }^{6}$ o el terrorismo islámico ${ }^{7}$, que afectan directa o indirectamente a la inmigración. Los propios medios han contribuido a ello, de forma especial con la representación realizada de los inmigrantes de origen árabe y su asociación con el islamismo. Por otra parte, la palabra ilegal es una de las más empleadas para referirse a las personas inmigrantes. El hecho de poner tanto énfasis en la asociación inmigranteilegal $^{8}$ estigmatiza a las personas de origen extranjero y las sitúa conceptualmente dentro del marco de lo delictivo, vinculándolas con la peligrosidad, la amenaza o la perturbación. Esto promueve la despersonalización y la criminalización injustificada del inmigrante y sirve como un fundamento más para el miedo y la prevención.

Editions de Fallois, 1990; HIXSON, W. Parting the curtain: propaganda, culture and the Cold War, 1945-1961, Basingstoke, Macmillan, 1997; SAUNDERS, F. La CLA y la guerra fría cultural, Madrid, Debate, 2001; NEVILLE, J. The press, the Rosenbergs, and the Cold War, Westport Praeger, 1995; SNYDER, A. Warriors of disinformation: American propaganda, Soviet lies, and the winning of the Cold War: an insider's account, New York, Arcade, cop, 1995.

"SEATON, J. "Las nuevas guerras "étnicas" y los medios de comunicación". En: Voces y Culturas. No 15. 2000, pp. 29-59. Op. Cit. en BAÑÓN HERNÁNDEZ, A. Discurso e inmigración. Propuestas para el análisis de un debate social, Murcia, Universidad de Murcia, 2002, p. 170.

7 Sobre la identificación del mundo islámico con el terrorismo, resulta de gran interés: CHOMSKY, Noam. La segunda guerra fría: crítica de la política exterior norteamericana, sus mitos y su propaganda, Barcelona, Crítica, 1984; acerca de la "islamización" del terrorismo internacional: GIORDANO, Eduardo. "El discurso periodístico sobre terrorismo “islámico" y la promoción del rearme”. En: Voces y Cultura. N. . 15, 2000, pp. 77-88.

${ }^{8} \mathrm{La}$ asociación de la inmigración con la marginalidad y la delincuencia ha sido puesta de manifiesto por, entre otros: CASERO RIPOLLÉS, A. "Discurso mediático, inmigración e ilegalidad: legitimar la exclusión a través de la noticia". En: ZAPATA BARRERO, R. y VAN DIJK, Teun A. (Eds.). Discursos sobre la inmigración en España. Los medios de comunicación, los parlamentos y las administraciones, Barcelona, Fundación CIDOB, 2007, pp. 69-90. 
La selección tendenciosa de situaciones, anécdotas, ejemplos y testimonios puede contribuir a la reproducción de numerosos tópicos y prejuicios. En ocasiones se intensifica la idea de inactividad o ausencia de interés por parte de los grupos marginales como causa de su situación. De este modo, se les presenta como los responsables únicos de la misma y se elude hablar de otros condicionantes ajenos que hayan podido influir. Otro de los recursos más empleados es el de presentar a las personas marginadas como seres que no distinguen entre lo imprescindible y lo superfluo, que se aprovechan de las coberturas públicas, que dilapidan sus recursos, o que prefieren dedicarse al ocio antes que cubrir sus necesidades básicas ${ }^{9}$.

Todo ello son modos, estrategias, herramientas que se emplean con demasiada frecuencia para mermar la carga reivindicativa del discurso de las personas inmigrantes, situar al "ellos" en posiciones deslegitimadas y hacer una representación del "nosotros" en la que se nos descarga de toda responsabilidad en estos procesos. De este modo, la imagen que se proyecta del discurso reivindicativo queda sustancialmente desdibujada, se le aplica una reducción importante y se le hace perder una parte sustancial de todo su potencial movilizador.

El discurso del compromiso está elaborado desde el "nosotros", miembros del endogrupo que se interesan por los procesos y las situaciones derivadas del fenómeno migratorio, que se manifiestan y

${ }^{9}$ Sirva para ilustrarlo el ejemplo de Bañón a partir de un artículo publicado en El Mundo en febrero de 2000: En ese mismo contexto [los dramáticos disturbios de El Ejido], el 26 de febrero leemos: "Firmada una paz momentánea, el pueblo sigue haciendo su vida. Es el caso de Karim, que no ha tocado una mata de pimientos desde que estalló el aquelarre racista. Es el caso de Ahmed, que perdió su tesoro más preciado: un transistor donde escuchar los goles del Deportivo de La Coruña». ¿Dijo Abmed que el transistor era su tesoro más preciado o lo interpreta el periodista?

SIMÓN, Pedro. "Los inmigrantes amplian el plazo de suspensión de la buelga". El Mundo, 26 de febrero de 2000, p. 39.

En: BAÑÓN HERNÁNDEZ, Antonio M. Discurso e inmigración. Propuestas para el análisis de un debate social. Murcia, Universidad de Murcia, 2002, Op. Cit., p. 185. 
actúan para responder a las necesidades de las personas inmigrantes y que tratan de contribuir a su integración en la sociedad española. La representación que de ello hace la prensa no deja de condicionar el mensaje y la imagen que se transmite, tanto del discurso propiamente como de sus emisores. La primera característica especialmente significativa es la imagen hiperbólica del compromiso en España. Como se ha dicho previamente, la mayor parte de los profesionales que trabajan en los principales medios de prensa y que tratan el tema de la inmigración no forman parte de colectivos inmigrante, sino que suelen ser españoles pertenecientes a un nivel sociocultural medio-alto. De este modo, conforme a la autorrepresentación positiva de la que nos habla Van Dijk, resulta natural que tiendan remarcar la imagen positiva del colectivo autóctono. Esto se percibe con especial claridad cuando se tratan temas como la solidaridad española con el Tercer Mundo, la ayuda a los refugiados o el compromiso hacia las minorías étnicas.

La manipulación de los tonos del compromiso puede dar lugar a diferentes interpretaciones sobre la intensidad de lo que se enuncia. El tono del discurso comprometido puede elevarse o rebajarse con diversos recursos. Por ejemplo, cuando se cambia selectivamente un verbo por otro: reclamar, pedir, hacer un llamamiento, denunciar, exigir, solicitar, requerir, instar, etc. También cuando se reduce o no se menciona en el titular lo que se pide o se solicita para las personas inmigrantes.

Actos como un comunicado, una rueda de prensa, una manifestación o un encierro suelen llevarse a cabo conjuntamente por agentes reivindicadores y por agentes comprometidos. De este modo, se aumentan los participantes, se unifican los discursos y se le da al acto en sí una mayor capacidad de persuasión. Se logra una mayor sensibilización social si la denuncia de una discriminación o la reivindicación de un derecho determinado no proviene sólo de personas del exogrupo, sino que también es obra de miembros pertenecientes al endogrupo. Sin embargo, en ocasiones se omite esta corresponsabilidad discursiva apartando de su autoría a uno de los dos agentes: bien al agente comprometido, restando 
importancia al componente de compromiso proveniente del endogrupo mayoritario; o bien al agente reivindicativo, reduciendo el valor del componente reivindicativo del mensaje enunciado.

El empleo del estilo indirecto puede dar lugar a variaciones semánticas que modifiquen la apariencia de las valoraciones realizadas por los actores comprometidos acerca de la inmigración, las personas inmigrantes o sus planteamientos. De este modo, se les pueden adjudicar palabras, expresiones y razonamientos que no han enunciado, lo que proyecta una imagen deslegitimada en mayor o en menor medida.

El discurso discriminatorio ${ }^{10}$ es la cara opuesta al discurso del compromiso. Ambos se formulan desde el ámbito del "nosotros". Sus portadores son miembros del endogrupo dominante. Sin embargo, uno y otro son el reflejo de universos mentales y concepciones del ser humano y las relaciones intergrupales muy diferentes. En el tratamiento que realiza la prensa de este discurso, se perciben igualmente ciertas carencias denunciadas por Bañón que es preciso conocer. El discurso discriminatorio encuentra en ocasiones dentro de los medios una plataforma que le permite despertar interés y obtener publicidad gratuita, lo que al margen de las valoraciones obtenidas, es considerado como un auténtico éxito por parte de sus impulsores.

En ocasiones se presentan las noticias de una manera que parece que existan una serie de realidades objetivas como pueden ser la crisis económica, el paro, el crecimiento de la inmigración o el aumento de la inseguridad ciudadana, que tienen como consecuencia reacciones violentas ejercidas por minorías de grupos vinculados a posiciones ideológicas de

10 Sobre el discurso discriminatorio y racista, véanse: VAN DIJK, Teun A. Racismo y análisis crítico de los medios, Barcelona, Paidós, 1997; BAÑÓN HERNÁNDEZ, A. Racismo, discurso periodístico y didáctica de la lengua. Almería, Universidad de Almería, 1996; CALVO BUEZAS, Tomás. Inmigración y racismo: asi sienten los jóvenes del siglo XXI. Madrid, Ed. Cauce, 2000; DÍEZ NICOLÁS, Juan. Los españoles y la inmigración, Madrid, Observatorio Permanente de la Inmigración, 1999. 
extrema derecha. En este sentido, la justificación, por medio de la elusión o de la atenuación discursiva, de las agresiones racistas y xenófobas es un caso emblemático de este tipo de valoración no negativa del comportamiento discriminatorio ${ }^{11}$. Para provocar esta atenuación existen fórmulas como relacionar una agresión con que el agredido tuviera antecedentes; representar con especial importancia las declaraciones de otros miembros del endogrupo que destaquen las cualidades del agresor en su comportamiento cotidiano, atención que no suele darse a los miembros del exogrupo; o incluso presentar a agresores como perturbados mentales, como jóvenes desorientados o como minorías marginales.

Por último, existe un concepto, el "ajuste de cuentas", utilizado de manera a veces exagerada para catalogar determinados acontecimientos violentos, que no siempre se corresponde con la realidad de lo sucedido y que produce el pernicioso efecto de la criminalización gratuita de la persona inmigrante $y$, por extensión, todo el colectivo. El "ajuste de cuentas", que se emplea mucho en actos violentos vinculados a la delincuencia, implica que antes de la agresión ha habido un comportamiento no adecuado por parte del agredido, lo cual no siempre es verdad. Se trata de una expresión que debe usarse con mucha prudencia si no se quiere caer en la difamación. Sin embargo, en muchas ocasiones, se emplea como una fórmula casi automática que deforma la realidad y que tiene el a veces perturbador y pernicioso efecto de identificar al agresor y al agredido como miembros de una misma categoría.

Todas estas son características que pueden apreciarse si se analiza de manera crítica el discurso periodístico sobre inmigración en España. Dentro de una valoración global, de conjunto, se perciben dos grupos prioritarios: por un lado "nosotros", el endogrupo, la sociedad autóctona de acogida; y por otro lado "ellos", el exogrupo, el colectivo formado por todas las personas inmigrantes. El tratamiento que "ellos" reciben por

${ }^{11}$ BAÑÓN HERNÁNDEZ, Antonio M. Discurso e inmigración... 2002, Op. Cit., p. 267. 
parte de la prensa española tiene, como se ha dicho, una serie de elementos comunes en mayor o menor medida. Sin embargo, si nos paramos a observar el trato dispensado de forma diferenciada a los miembros de las distintas nacionalidades o, más bien, grupos étnicos que conforman el colectivo inmigrante, se aprecian también ciertas peculiaridades que pueden darse en mayor o menor medida en función de la nacionalidad de que se trate.

\section{ANÁLISIS EMPÍRICO DE PRENSA EN ESPAÑA: EL MUNDO Y EL PAÍS}

Zapata-Barrero plantea un análisis del marco discursivo político en España con respecto a la inmigración a partir de dos categorías: el discurso reactivo y el discurso proactivo. El discurso reactivo reacciona contra el proceso de cambio histórico hacia la multiculturalidad protagonizado por la inmigración, buscando restablecer el pasado monocultural; percibe la llegada de inmigrantes como algo negativo; y asume un marco de referencia poblacional en el que se da prioridad a la población ciudadana frente a los no ciudadanos. El discurso proactivo asume el proceso de cambio histórico hacia la multiculturalidad, tratando de contribuir a que éste se realice de manera normalizada y exitosa; percibe la llegada de inmigrantes como una oportunidad y un reto que hay que afrontar; y toma un marco de referencia en el que se tienen en cuenta los intereses de toda la población, al margen de que sea población ciudadana o no ${ }^{12}$.

El autor del presente artículo, realizó en su Tesis Doctoral un análisis exhaustivo del discurso periodístico en España con respecto a la inmigración y a la diversidad cultural. Para ello, analizó las noticias

12 Véase: ZAPATA-BARRERO, Ricard. "La política del discurso sobre la inmigración en España”. En: ZAPATA-BARRERO, Ricard, GONZÁLEZ, Elisabet y SÁNCHEZ MONTIJANO, Elena. El discurso politico en torno a la inmigración en España y en la Unión Europea, Madrid, Ministerio de Trabajo e Inmigración. 2008, pp. 117-157. 
publicadas dentro de esta temática por los dos periódicos con más tirada e impacto en España, los diarios El Mundo y El País entre los años 2008 y 2010, que fueron los años en los que el discurso político en torno a la inmigración viró desde una posición más sensible con la situación del colectivo inmigrante hacia unas políticas más restrictivas. Este trabajo, publicado en $2012^{13}$, muestra de forma palpable que el discurso periodístico concede una gran importancia a asuntos como la inmigración irregular, la economía sumergida o la delincuencia, aspectos que devalúan o criminalizan la imagen de la persona inmigrante, $y$, sin embargo, le presta una atención menor a la denuncia de las restricciones a los derechos de las personas inmigrantes $y$, en especial, los trabajadores. Se aprecia contraste entre la receptividad que muestra la prensa nacional a los aspectos negativos referidos a la inmigración y los aspectos que ofrecen una visión más positiva de ella o a los agentes reivindicativos y comprometidos.

Se perciben rasgos comunes en los discursos de los medios elegidos para el análisis de prensa y también determinados elementos divergentes que afloran como producto de líneas editoriales distintas. Así, con matices y salvedades, se aprecia en El Mundo, como es sabido cercano al $\mathrm{PP}^{14}$, una mayor cobertura del discurso que tiende a hiperbolizar el volumen de la inmigración; reproduce en mayor medida noticias, acontecimientos, sucesos, manifestaciones, que ofrecen una representación del inmigrante asociada a la ilegalidad e incluso a la criminalidad ocasionalmente; se hace eco de las protestas contra la Reforma de la Ley de Extranjería ${ }^{15}$ y contra la ampliación de la permanencia en los Centros de

${ }^{13}$ GARRIDO RODRÍGUEZ, Pedro. Inmigración y diversidad cultural en España. Un análisis bistórico desde la perspectiva de los Derechos Humanos, Salamanca, Ediciones Universidad de Salamanca, Colección Vítor, 2012.

${ }^{14}$ Partido hegemónico del centroderecha en la democracia española.

${ }^{15}$ JEFATURA DEL ESTADO. Ley Orgánica 2/2009, de 11 de diciembre, de reforma de la Ley Orgánica 4/2000, de 11 de enero, sobre derechos y libertades de los extranjeros en España y su integración social. BOE No 299, de 12 de diciembre de 2009. 
Internamiento de Extranjeros ${ }^{16}$; refleja en tono neutro o positivo el cambio de discurso del PSOE $^{17}$ en materia de inmigración, más endurecido, a fin de subrayar su confluencia con las posiciones del PP en algunos de sus presupuestos, durante la segunda legislatura; es más explícito e insiste en mayor medida en las divergencias entre los líderes del PSOE en materia migratoria; apoya o es más favorable al paradigma europeo de gestión de la inmigración, que hace hincapié en el control de flujos migratorios y apuesta por una inmigración selectiva en el ámbito laboral, en la línea del PP y de países referencia en Europa como Francia y Alemania; recoge noticias en las que se ponen de manifiesto las aportaciones de los inmigrantes al sistema productivo y al Estado del Bienestar. Dentro del ámbito educativo, El Mundo incide con frecuencia en las repercusiones negativas del alumnado inmigrante y en sus dificultades de integración; critica la concentración de alumnado inmigrante en determinados centros y, sin embargo, se muestra receloso ante la posibilidad de segregar a alumnos extranjeros recién llegados en centros especiales, como ha sido planteado y puesto en práctica en Cataluña; se hace eco de buenas prácticas en educación intercultural. En lo relativo a la diversidad cultural, la política de "una de cal y otra de arena" le lleva a dar cabida a opiniones de diversa índole, incluyendo las de personas procedentes de otras culturas que explican sus identidades culturales y comparten experiencias; recoge las explicaciones de expertos, estudiosos y personas pertenecientes a otras culturas, que explican sus identidades culturales, desmienten falsas creencias y comparten

16 La Ley 2/2009 amplía el plazo máximo de permanencia en los Centro de Internamiento de Extranjeros (CIE), en los que las personas inmigrantes en situación irregular son internados cuando mientras se estudia su expediente de expulsión. El hecho de que una infracción administrativa, como es el hecho de no estar regularizado, implique el internamiento en este tipo de centros ha sido duramente criticado por múltiples asociaciones y ONG.

${ }^{17}$ Partido principal del centroizquierda en España. 
experiencias; reproduce y celebra ejemplos de buenas prácticas interculturales; se muestra tolerante o critica de forma laxa puntualmente iniciativas como los contratos del integración, propuestos por el PP a semejanza de los contratos impulsados en Francia.

En El País, afín al PSOE como es conocido, se aprecia también, según y cuando, la presencia de un discurso que tiende a hiperbolizar el volumen de la inmigración. Ocasionalmente se percibe, aunque en menor medida que en El Mundo, la difusión de noticias, acontecimientos, sucesos y manifestaciones que ofrecen una representación del inmigrante asociada a la ilegalidad o a la criminalidad. Por otro lado, en un afán por aparecer como medio independiente, en consonancia con una imagen buscada de centro-izquierda, reproduce y se suma a las críticas contra la Reforma de la Ley de Extranjería y muy especialmente contra los Centros de Internamiento de Extranjeros y la ampliación del plazo de permanencia en ellos; critica duramente iniciativas como los contratos de integración para extranjeros, tanto en España como en Europa; se muestra más crítico que El Mundo con los cambios de posicionamiento del PSOE con respecto a la inmigración y su acercamiento al PP en la segunda legislatura y a la hora de tratar las divergencias entre los líderes políticos del PSOE en cuestiones de inmigración, es menos incisivo que El Mundo. Critica, sin embargo, aquellos aspectos del Derecho Migratorio Europeo que considera que menoscaban los derechos de los inmigrantes, especialmente en cuestiones como la Directiva del Retorno o la Directiva de la Tarjeta Azul; da amplia cabida a noticias que explicitan las aportaciones de los inmigrantes al sistema productivo y al Estado del Bienestar, ofreciendo una visión amplia de la inmigración y sus implicaciones y desmontando falsas creencias. Dentro del ámbito educativo, recoge datos que hablan de una mayor tasa de fracaso escolar por parte del alumnado inmigrante y de un impacto negativo en las estadísticas globales de fracaso escolar, pero no lo asocia tanto al propio alumnado en sí, sino que lo relaciona más bien con fenómenos de gestión como la concentración del alumnado inmigrante, la guetización en determinados centros y las carencias del sistema educativo 
para dar respuesta a la nueva realidad pluricultural; critica iniciativas como la segregación escolar de alumnos extranjeros recién llegados en Espacios de Bienvenida Educativa, pese a ser defendidos por algunos líderes del PSOE, como el entonces Ministro de Trabajo e Inmigración, Celestino Corbacho y se hace eco y celebra buenas prácticas en interculturalidad. Dentro del terreno de la diversidad cultural, critica la politización de asuntos delicados como el velo, en línea con la entonces Vicepresidenta del Gobierno del PSOE, M ${ }^{\mathrm{a}}$ Teresa Fernández de la Vega; reproduce las aportaciones de personas de otras culturas que explican determinados aspectos referidos a su identidad cultural y comparten experiencias y, sobre todo, recoge las explicaciones de expertos y estudiosos pertenecientes a diversas culturas que tratan de clarificar aspectos relacionados con identidades culturales diversas, desmienten falsas creencias y comparten experiencias; recoge en mayor medida que El Mundo estudios, investigaciones, acontecimientos, opiniones, testimonios que ofrecen una visión más conciliadora entre el Islam y las democracias occidentales y el Discurso Internacional de los Derechos Humanos.

Adicionalmente a estas diferencias sustanciosas en el tratamiento de la inmigración en los diarios El Mundo y El País, podemos apreciar determinados rasgos comunes en los que se materializan los discursos reactivo y proactivo. Vemos cómo se reproduce ampliamente el discurso de las élites políticas, como ha sido puesto de manifiesto por autores como Van Dijk. Existe una gran cobertura mediática de sus acuerdos, sus debates, sus leyes, etc., pero no se le da una importancia proporcional a las reivindicaciones protagonizadas por asociaciones de inmigrantes y ONG, que en realidad son también protagonistas de los procesos derivados de la inmigración, así como destinatarios directos de los acuerdos tomados en la esfera política, pero a los que sólo se da voz de modo ocasional.

A lo largo del análisis de prensa realizado, percibimos la presencia ambivalente de dos discursos. Uno es más bien reactivo o receloso con respecto a la inmigración y sus diversas implicaciones políticas, 
socioeconómicas y culturales y el otro muestra un carácter más proactivo, tolerante o favorable a estos procesos ${ }^{18}$.

El discurso reactivo se caracteriza en lo que concierne a la recepción de los inmigrantes por conceder una mayor importancia a los aspectos más negativos como la entrada irregular, las conexiones con las mafias, la delincuencia o la inseguridad ciudadana, casi siempre vistos desde los ojos del endogrupo, así como el empleo de términos exagerados, despectivos o con connotaciones innecesariamente negativas para referirse a las personas inmigrantes. Hace especial hincapié en el empleo irregular, la economía sumergida, los fraudes, la denuncia de estas cuestiones como una maniobra de autorrepresentación positiva, la presentación de los inmigrantes como consumidores de recursos sociales y la minimización de la importancia de la contribución de los trabajadores inmigrantes a la Seguridad Social. Con frecuencia se subrayan los aspectos más problemáticos de la heterogeneidad de culturas y de una sociedad multicultural, en lugar de sus potencialidades y se presenta a determinadas identidades culturales como escasamente compatibles con una sociedad democrática como la nuestra. Esto sucede especialmente con la comunidad islámica. Encontramos numerosas noticias, artículos, reportajes y columnas de opinión en las que se resaltan los preceptos, planteamientos y acciones más retrógrados que se llevan a cabo, bajo el argumento de que la religión islámica lo exige. En ocasiones, vemos que cuando se denuncian casos en los que en nombre de la cultura se vulneran los derechos culturales e incluso los Derechos Humanos de personas a las

${ }_{18}$ Como se ha dicho, se emplean los términos reactivo y proactivo en el análisis del discurso periodístico a semejanza de la terminología empleada por Zapata-Barrero en su análisis de los discursos políticos en materia de inmigración. Sobre ello, consúltese: ZAPATA-BARRERO, Ricard (2008): La política del discurso sobre la inmigración en España. En: ZAPATA-BARRERO, Ricard, GONZÁLEZ, Elisabet y SÁNCHEZ MONTIJANO, Elena. El discurso político en torno a la inmigración en España y en la Unión Europea, Madrid, Ministerio de Trabajo e Inmigración, 2008, Op. Cit., pp. 117-157. 
que se les niega la libertad para elegir, más que hacerse en defensa de estas personas, se utiliza el caso como argumento para representar negativamente a la identidad cultural en cuyo nombre se comete este abuso, poniendo poco énfasis en advertir que en este tipo de acciones confluyen en muchas ocasiones otro tipo de intereses más bien políticos, económicos y de poder, que en realidad nada tienen que ver con la cultura en sí, sino que ésta se ve utilizada como parangón para justificar dichas acciones. También es patente cómo en múltiples ocasiones se incurre en un reduccionismo nada objetivo a la hora de hacer valoraciones sobre las culturas del exogrupo. El ejemplo más notorio es quizá la identificación entre islamismo y fundamentalismo, cuando, si nos atenemos a la realidad, vemos que el fundamentalismo está más vinculado a intereses políticos y económicos que a la religión y a la cultura propiamente. Percibimos también que no es un fenómeno en el que esté implicado todo el conjunto de la población islámica, sino que se da más bien de manera bastante polarizada en determinados países donde este fundamentalismo, lejos de crecer de manera natural entre la población, es alentado interesadamente desde esferas de poder que no actúan motivadas por una creencia religiosa o cultural, sino movidas por intereses políticos y económicos. A su vez, se concede un espacio y una atención mucho menor a las solicitudes del movimiento asociativo así como a las iniciativas favorables a la integración en la sociedad española que parten de las propias comunidades de inmigrantes y que ponen de manifiesto su compromiso de formar parte activa de una sociedad multicultural basada en el intercambio y el mutuo enriquecimiento sobre la base del respeto a los valores democráticos y a los Derechos Humanos.

Como se ha dicho, el discurso mediático presenta también, como reflejo a su vez del discurso político, un carácter más proactivo, tolerante o favorable con respecto a la inmigración y sus implicaciones. Esto se manifiesta con publicaciones, aún minoritarias, con un enfoque más equilibrado de la inmigración en España y lo que representa. También la concesión de cierto espacio mediático a las acciones reivindicativas 
desempeñadas por las asociaciones de inmigrantes. Noticias críticas con la situación de los inmigrantes y con la respuesta dada por el sistema al drama de la inmigración irregular. Artículos en los que se reconoce la contribución de los trabajadores inmigrantes al Estado del Bienestar desde múltiples aspectos, especialmente por la contribución a la Seguridad Social y por el aumento de la tasa de natalidad. También percibimos la progresiva presencia de un enfoque proactivo o tolerante en la cuestión de la diversidad en España.

\section{PROPUESTAS DE BUENAS PRÁCTICAS EN EL TRATAMIENTO EN PRENSA DE LA INMIGRACIÓN Y LA DIVERSIDAD CULTURAL}

El tratamiento que realizan los medios de comunicación sobre la inmigración y sus numerosas implicaciones no solamente es analizado en el marco de la investigación académica. También existen importantes e ilustrativos trabajos realizados por organizaciones sociales, ONG y asociaciones de prensa. Entre estos numerosos y diversos estudios, informes, guías, etc., merece una atención especial, por su grado de pluralidad y consenso, por su trascendencia y por su nivel institucional, el documento Guia práctica para los profesionales de los medios de comunicación: tratamiento mediático de la inmigración ${ }^{19}$, dirigido y coordinado por el Observatorio Español del Racismo y la Xenofobia. La Guía, publicada en 2008, cuenta en su elaboración con un conjunto de colaboradores entre los que se integran representantes de diversos medios y agencias de comunicación, organizaciones sociales, ONG y asociaciones de personas

${ }^{19}$ SENDÍN GUTIÉRREZ, José C. e IZQUIERDO IRANZO, Patricia. Guia práctica para los profesionales de los medios de comunicación: tratamiento mediático de la inmigración, Madrid, Ed. Subdirección General de Información Administrativa y Publicaciones. Ministerio de Trabajo y Asuntos Sociales, 2008. 
inmigrantes ${ }^{20}$. Toma también la referencia del Programa de Acción Comunitaria de lucha contra la discriminación (2001-2006) ${ }^{21}$, dirigido a todos aquellos agentes implicados que pudieran ayudar a desarrollar políticas y normativa adecuada y eficiente para eliminar la discriminación. El Programa plantea tres objetivos prioritarios: mejorar el entendimiento de materias relacionadas con la discriminación; desarrollar la capacidad de abordar de forma efectiva la discriminación; y promover los valores que subyacen a la lucha contra la discriminación. La Guía, como queda expuesto en el primer bloque de la misma, dentro del apartado dedicado a los retos de los profesionales de los medios de comunicación, se crea con el objetivo de ser un instrumento de ayuda consensuado con los profesionales de los

${ }^{20}$ La autoría de la Guía práctica para los profesionales de los medios de comunicación: tratamiento mediático de la inmigración corre a cargo de José C. Sendín Gutiérrez y Patricia Izquierdo Iranzo, del Grupo de investigación sobre comunicación sociedad y cultura (GICOMSOC), de la Universidad Rey Juan Carlos. En cuanto al grupo de colaboradores para su elaboración, está compuesto por: Ana Rodrigo (Agencia EFE), María Pin Agencia (EUROPA PRESS), Julio García (Agencias SERVIMEDIA), Abigail Campos (Agencia FAX PRESS), Cruz Morcillo (ABC), David Gracia (Expansión), Luis Izquierdo (La Vanguardia), Eva Revenga (COPE), Jordi Ferrerons (CUATRO), Minerva Oso (RNE), Nicolás Castellano (SER), Patricia Villarruel (El Universo de Ecuador y Revista “Raíz”), Félix Damián Roman (In Lume), Soraya Constante (Semanario Latino), Angel Sabat Diario (Sí se puede), Africa Martínez (Pueblo Nuevo), Gabriela Puerto Revista (Lazo Latino), Itziar Marañón Revista (Toumai).

21 CONSEJO DE LA UNIÓN EUROPEA. Decisión 2000/750/CE del Consejo de 27 de noviembre de 2000 por la que se establece un programa de acción comunitario para luchar contra la discriminación (2001-2006). Diario Oficial de las Comunidades Europeas L 303/23, de 2 de diciembre de 2000. Para la consecución de los objetivos, se plantean tres acciones transnacionales:

- Análisis de los factores relacionados con la discriminación (recopilación de estadísticas, estudios, evaluación de la eficacia de las políticas y difusión de los resultados).

- Cooperación transnacional entre los agentes específicos y creación de redes europeas de ONG;

- Sensibilización sobre la dimensión europea de la lucha contra la discriminación. 
DELICTAE, Vol. 4, No6, Jan..-Jun. $2019 \mid 26$

medios, tanto generalistas, como dirigidos al colectivo de inmigrantes, con el fin de ofrecer pautas de comprensión y tratamiento de este fenómeno de la inmigración, tan reciente en nuestro país como normal a lo largo de la historia de España, sobre todo en el último siglo ${ }^{22}$.

El fundamento de este compromiso es la responsabilidad social que tienen los profesionales de la comunicación dada la capacidad que atesoran los contenidos mediáticos y su puesta en escena para influir en las audiencias $^{23}$. En este sentido, se afirma que el desarrollo de la actividad periodística ha evolucionado en gran medida en los últimos años, pasando de una perspectiva única, centrada en resaltar lo negativo y lo pintoresco de la inmigración y en reproducir los estereotipos creados por el endogrupo, a un enfoque cada vez más plural en el que se contempla una mayor diversidad de puntos de vista. No obstante, también han sido denunciados, por otra parte, ciertos procesos y rutinas profesionales influidos por un contexto social eurocéntrico que hacen que se reproduzcan velada, tácita o incluso inconscientemente, sutiles manifestaciones de racismo discursivo ${ }^{24}$.

La Guía contiene un estado de la cuestión, un análisis de buenas y malas prácticas y una serie de recomendaciones. Para ello no parte de cero. Recoge los frutos de la experiencia de amplios y diversos trabajos previos llevados a cabo por diferentes asociaciones profesionales periodísticas, así como instituciones públicas especializadas en la cobertura y reproducción de la inmigración en los medios de comunicación ${ }^{25}$. Entre todos estos

22 SENDÍN GUTIÉRREZ, José C. e IZQUIERDO IRANZO, Patricia. Guia práctica para los profesionales de los medios de comunicación... 2008, Op. Cit., p. 10.

23 Particularmente se han ocupado de estudiar el fenómeno teorías contrastadas como la de la fijación de la agenda (agenda-setting) o la de la creación de marcos interpretativos o encuadres noticiosos (framing).

${ }^{24}$ VAN DIJK, Teun A. Racismo y análisis crítico de los medios... 1997, Op. Cit.

${ }^{25}$ En este sentido, son especialmente reseñables los Consejos Audiovisuales de Cataluña (CAC), Navarra (COAN) y Andalucía (CAA); así como asociaciones profesionales de periodistas, como la Federación de Asociaciones de la Prensa de España (FAPE), y la 
trabajos, españoles y europeos, acerca del correcto tratamiento mediático de la inmigración, resultan de especial importancia: Manual de estilo sobre minorías étnicas, elaborado por el Colegio de Periodistas de Cataluña ${ }^{26}$; Recomendaciones del Consejo del Audiovisual de Cataluña sobre el Tratamiento

Federación Andaluza de Asociaciones de la Prensa (FAAP). Para un conocimiento más profundo de las propuestas más relevantes sobre, también resulta muy ilustrativo el documento: Inmigración y Medios de Comunicación. Manual recopilatorio de buenas prácticas periodísticas, elaborado por la Coordinadora de ONG de Euskadi de apoyo a inmigrantes. Sus materiales también han servido de gran ayuda para la elaboración de este apartado. Puede consultarse en el sitio web: http://www.mugak.eu/gunea/obsmedios/buenpract/ [21/07/2016].

${ }^{26}$ COLEGIO DE PERIODISTAS DE CATALUÑA. "Manual de estilo sobre minorías étnicas". En: Quaderns del CAC. Número 12. Enero-Abril de 2002. Pp. 68-70. Disponible en: www.audiovisualcat.net [21/07/2016]. El Manual de estilo sobre minorías étnicas se basa en siete principios fundamentales:

1) No hay que incluir el grupo étnico, el color de la piel, el país de origen, la religión o la cultura si no es estrictamente necesario para la comprensión global de la noticia.

2) Es necesario evitar las generalidades, los maniqueísmos y la simplificación de las informaciones. Los residentes extranjeros no comunitarios son tan poco homogéneos como los autóctonos.

3) No deben potenciarse las informaciones negativas ni las sensacionalistas. Hay que evitar crear inútilmente conflictos y dramatizarlos. Hay que potenciar la búsqueda de noticias positivas.

4) Ecuanimidad en las fuentes de información. Es necesario contrastar las versiones institucionales. Hay que potenciar las propias de las minorías étnicas y tener especial cuidado en las informaciones referidas a los países de origen. La publicación de las rectificaciones como elementos que inciden en la calidad del medio informativo.

5) Responsabilidad de los profesionales. La importancia de la ubicación física de la información. "El efecto dominó". Utilización del material gráfico.

6) Militancia periodística: hacia una multi-interculturidad enriquecedora para todos. La potenciación de las informaciones en positivo.

7) El uso del género gramatical masculino para referirse a grupos mixtos invisibiliza a las mujeres y sus aportaciones en los diferentes ámbitos de vida, simplificando y, en muchos casos, deformando la realidad. 
Informativo de la inmigración ${ }^{27}$; Recomendaciones para evitar la invisibilización de las mujeres inmigrantes y su victimización en las informaciones, del Centro de Estudios y Documentación sobre Inmigración, Racismo y Xenofobia (MUGAK) de SOS Racismo ${ }^{28}$; Manual para los periodistas sobre la protección de la infancia en los medios de comunicación, de la Unió de Periodistes Valencians ${ }^{29}$; Racism and Cultural Diversity in the Mass Media. An overview of research and examples of good practice in the EU Member States, del Observatorio Europeo del Racismo y la Xenofobia (EUMC) ${ }^{30}$; Seminario Euromediterráneo Racism, Xenophobia and

27 CONSEJO AUDIOVISUAL DE CATALUÑA. "Recomendaciones del Consejo del Audiovisual de Cataluña sobre el Tratamiento Informativo de la Inmigración". En: Quaderns del CAC, Número 12. Enero-Abril de 2002, pp: 63-67. A disposición en www.audiovisualcat.net [21/07/2016]. Las recomendaciones del Consejo Audiovisual de Cataluña se clasifican en cuatro áreas: recomendaciones dirigidas a las autoridades; recomendaciones dirigidas a las empresas audiovisuales; recomendaciones dirigidas a los profesionales de la información audiovisual; y recomendaciones de carácter general.

28 PÉREZ WOLFRAM, Clara. "Las inmigrantes en la prensa: víctimas sin proyecto migratorio". En revista: MUGAK. N ${ }^{\circ} 24$, Centro de Estudios y Documentación sobre Inmigración, Racismo y Xenofobia (MUGAK) de SOS Racismo, 2005. Disponible en: http://revista.mugak.eu/articulos/ [21/07/2016]. Tras un completo estado de la cuestión, se proponen en este artículo una serie de recomendaciones para evitar la invisibilización de las mujeres inmigrantes y su victimización en las informaciones.

${ }^{29} \mathrm{El}$ Manual para los periodistas sobre la protección de la infancia en los medios de comunicación, elaborado por la Unió de Periodistes Valencians, realiza una serie de orientaciones para el tratamiento informativo de menores que pueden aplicarse al caso concreto de menores de origen inmigrante. Para mayor información, puede consultarse: http://www.unioperiodistes.org [27/08/2019].

30 OBSERVATORIO EUROPEO DEL RACISMO Y LA XENOFOBIA (EUMC) y WAL, Jessica ter (Ed.). Racism and Cultural Diversity in the Mass Media. An overview of research and examples of good practice in the EU Member States 1995-2000, Viena, European Research Centre on Migration and Ethnic Relations (ERCOMER), 2002. El estudio analiza el modo en que se está tratando la inmigración y la diversidad cultural en los medios de diferentes países europeos (Bélgica, Dinamarca, Alemania, Grecia, España, Francia, Irlanda, Italia, Luxemburgo, Holanda, Austria, Portugal, Finlandia, Suecia y Reino Unido). Por otra parte, realiza diversas recomendaciones. En primer lugar, en el campo de la investigación y de los observatorios, sugiere que se debería poner mayor énfasis en 
the Media. Towards respect and understanding of all religions and cultures. EuroPartnership (2006) ${ }^{31}$.

Tomando como referencia todos estos trabajos previos, la Guía Práctica para los Profesionales de los Medios de Comunicación. Tratamiento de la

los aspectos relativos a la producción de la información (acceso y participación de los inmigrantes, formación de comunicadores, códigos profesionales, etc.); y en la recepción de los mensajes. En lo referido a los profesionales de los medios de comunicación, las recomendaciones se centran en el empleo de las fuentes y del lenguaje en la información política, para lo que propone tener ofrecer la palabra a actores con posiciones diversas y mantener un mayor control sobre el lenguaje empleado por los políticos y las fuentes oficiales, criticando claramente los prejuicios que pudiera contener; la búsqueda de la diversidad de fuentes, pasando de "hablar sobre inmigración" a hablar "con los inmigrantes"; y la promoción de programas e iniciativas de periodismo de investigación que ofrezcan un mayor conocimiento del origen, el contexto vital y la historia de las personas inmigrantes. Por último, se realizan recomendaciones para promover la diversidad, entre las que se encuentran la de apoyar la formación orientada a los inmigrantes, para que se incorporen a los medios de comunicación, mediante becas y acuerdos de colaboración; así como a los autóctonos, para que se sensibilicen con el tema y sean conscientes de su responsabilidad. En este sentido, también se propone favorecer el intercambio de información, la cooperación y la autorregulación en los medios de comunicación. Disponible en: http://fra.europa.eu/fraWebsite/ [27/08/2019].

31 En cuanto al seminario euromediterráneo Racism, Xenophobia and the Media. Towards respect and understanding of all religions and cultures. Euro-Partnership (2006), recoge las aportaciones de los miembros del acuerdo Euromediterráneo (25 de la Unión Europea y 10 socios más del Mediterráneo: Argelia, Egipto, Israel, Jordania, Líbano, Marruecos, Autoridad Palestina, Siria, Túnez y Turquía, más Libia con estatus de observador desde 1999). Establece recomendaciones por áreas, entre las cuales destacan recomendaciones dirigidas a las empresas mediáticas, recomendaciones dirigidas a las instituciones de formación de profesionales de los medios, así como a instituciones profesionales, recomendaciones dirigidas a la sociedad civil y grupos minoritarios y recomendaciones dirigidas a instituciones internacionales y europeas.

Las conclusiones del Seminario Euromediterráneo se encuentran disponibles en: http://fra.europa.eu/fra/material/pub/general/euromed conference report 220506 en.pdf o en: http://www.euromedalex.org/sites/default/files/euromed en.pdf [21/07/2016]. 
DELICTAE, Vol. 4, No6, Jan..-Jun. $2019 \mid \mathbf{3 0}$

Inmigración ${ }^{32}$ incluye unas recomendaciones propias que están orientadas hacia tres áreas primordiales: el colectivo de profesionales de los medios; las empresas de comunicación; y los centros de formación de profesionales de la comunicación y las instituciones públicas.

\section{CONCLUSIONES}

Los discursos político y mediático tienen un efecto en la sociedad que se percibe nítidamente. Existen múltiples consultas de opinión en las que podemos apreciar, según se ha dicho, que la percepción de la inmigración por parte de los españoles empeora a partir de 2008, coincidiendo con la situación de crisis económica, el viraje del discurso político y la modulación del discurso de prensa, que actúa como espejo de aquel. Se tiende a valorar a los inmigrantes en términos de problema, relacionándolos con la irregularidad, la criminalidad y la inseguridad, se hace un balance a la baja de sus contribuciones al país, se los ve más como competencia en el trabajo que como factor de crecimiento para el sistema productivo y se estiman en menor medida sus contribuciones, efectuadas o potenciales, al país. Todo ello hace patente el deterioro en la percepción acerca de los trabajadores foráneos y el recrudecimiento en los planeamientos ante la inmigración por parte de la sociedad española.

Por su parte, el colectivo inmigrante se ve incomodado ante esta situación y reclama sus derechos a través de actos reivindicativos, manifestaciones y actividades de protesta, como las acontecidas en 2009 a propósito de la Reforma de la Ley de Extranjería y la ampliación del plazo de permanencia en los Centros de Internamiento de Extranjeros. En ellos, las asociaciones de inmigrantes han contado normalmente con el apoyo de agentes sociales comprometidos, miembros de ONG e instituciones

32 SENDÍN GUTIÉRREZ, José C. e IZQUIERDO IRANZO, Patricia. Guia práctica para los profesionales de los medios de comunicación: tratamiento mediático de la inmigración. Madrid, Ed. Subdirección General de Información Administrativa y Publicaciones. Ministerio de Trabajo y Asuntos Sociales, 2008, Op. Cit. 
solidarias principalmente. Sin embargo, no siempre han tenido la cobertura mediática ni la receptividad política y social esperada.

El recrudecimiento del discurso político sobre inmigración, amparado a menudo en los medios, conlleva efectos perversos en nuestra sociedad. Puede contribuir a despertar actitudes recelosas ante la inmigración que sirven de caldo de cultivo para la aparición de grupos y movimientos de perfil discriminatorio. Esto es perceptible actualmente en la escena mundial, donde partidos y plataformas con posicionamientos abiertamente reactivos con las migraciones, cuando no estigmatizadores, xenófobos o manifiestamente racistas con las personas inmigrantes están encontrando un apoyo social inusitado.

Es crucial que esta clase de planteamientos ostensiblemente reaccionarios e incompatibles con el Estado de Derecho, lejos de encontrar cobijo en el desconocimiento de la realidad sociocultural, en el puro pragmatismo político y en la ausencia de valores de convivencia y solidaridad, generen rechazo y no tengan cabida en una sociedad moderna y democrática como la nuestra. Para lograr este fin, es necesario desarmar prejuicios, creencias erróneas y razonamientos no fundamentados en contra de la inmigración y de los inmigrantes, así como ofrecer un enfoque panorámico que integre todas las variables involucradas en la complejísima realidad de las migraciones. Solo así podrá prevalecer una gestión de las mismas que sea consecuente con el discurso positivo normativo internacional de los Derechos Humanos.

\section{REFERENCIAS BIBLIOGRÁFICAS}

ARON, R. Les articles de politique internationale dans le Figaro de 1947 à 1977, París, Editions de Fallois, 1990.

CASERO RIPOLLÉS, A. "Discurso mediático, inmigración e ilegalidad: legitimar la exclusión a través de la noticia”. En: ZAPATA BARRERO, R. y VAN DIJK, Teun A. (Eds.). Discursos sobre la inmigración en España. Los 
medios de comunicación, los parlamentos y las administraciones, Barcelona, Fundación CIDOB, 2007, pp. 69-90.

CHOMSKY, Noam. La segunda guerra fría: crítica de la politica exterior norteamericana, sus mitos y su propaganda, Barcelona, Crítica, 1984.

DÍEZ NICOLÁS, Juan. Los españoles y la inmigración, Madrid, Observatorio Permanente de la Inmigración, 1999.

GADDIS, J. L. La guerra fría, Barcelona, RBA, 2008.

GARRIDO RODRÍGUEZ, Pedro. Inmigración y diversidad cultural en España. Su gestión desde la bonanza económica a la crisis, Madrid, Ed. Fundamentos, 2014.

GARRIDO RODRÍGUEZ, Pedro. Inmigración y diversidad cultural en España. Un análisis histórico desde la perspectiva de los Derechos Humanos, Salamanca, Ediciones Universidad de Salamanca, Colección Vítor, 2012.

GIORDANO, Eduardo. "El discurso periodístico sobre terrorismo "islámico" y la promoción del rearme”. En: Voces y Cultura. N. ${ }^{\circ}$ 15, 2000, pp. 77-88.

HIXSON, W. Parting the curtain: propaganda, culture and the Cold War, 1945 1961, Basingstoke, Macmillan, 1997.

LAFEBER, W. America, Russia, and the cold war, McGraw-Hill, 1987.

MARTÍNEZ QUINTEIRO, M. ${ }^{a}$ Esther. "El discurso de los derechos humanos en perspectiva histórica. El síndrome de la torre de Babel”. En: PANDO BALLESTEROS, $M^{\mathrm{a}}$ Paz; GARRIDO RODRÍGUEZ, Pedro y MUÑOZ RAMÍREZ, Alicia (Eds.). El cincuentenario de los Pactos 
Internacionales de Derechos Humanos de la ONU. Homenaje a la Profesora $M^{a}$. Esther Martinez Quinteiro, Salamanca, Ediciones Universidad de Salamanca, Colección Aquilafuente, No 243, 2018, pp. 79-106.

NEVILLE, J. The press, the Rosenbergs, and the Cold War, Westport Praeger, 1995.

PANDO BALLESTEROS, María de la Paz; GARRIDO RODRÍGUEZ, Pedro y MUÑOZ RAMÍREZ, Alicia (Eds.). El cincuentenario de los Pactos Internacionales de Derechos Humanos de la ONU. Homenaje a la Profesora $M^{a}$. Esther Martinez Quinteiro, Salamanca, Ediciones Universidad de Salamanca, Colección Aquilafuente, No $^{\circ}$ 243, 2018.

PANDO BALLESTEROS, María de la Paz; MUÑOZ RAMÍREZ, Alicia y GARRIDO RODRÍGUEZ, Pedro (dirs. y eds.). Pasado y Presente de los Derechos Humanos. Mirando al Futuro. Madrid, Editorial Los Libros de la Catarata, 2016.

SAUNDERS, F. La CIA y la guerra fría cultural, Madrid, Debate, 2001.

SEATON, J. "Las nuevas guerras "étnicas" y los medios de comunicación”. En: Voces y Culturas. No 15. 2000, pp. 29-59.

SENDÍN GUTIÉRREZ, José C. e IZQUIERDO IRANZO, Patricia. Guía práctica para los profesionales de los medios de comunicación: tratamiento mediático de la inmigración, Madrid, Ed. Subdirección General de Información Administrativa y Publicaciones. Ministerio de Trabajo y Asuntos Sociales, 2008.

SNYDER, A. Warriors of disinformation: American propaganda, Soviet lies, and the winning of the Cold War: an insider's account, New York, Arcade, cop, 1995. 
VAN DIJK, Teun. "Discurso de las élites y racismo institucional". En: Bastida, L. (Coord.). Medios de comunicación e inmigración, Murcia, Caja de Ahorros del Mediterráneo-Obra Social, 2006, pp. 15-34.

VEIGA, F. y DUARTE, A. La paz simulada: una bistoria de la Guerra Fría 1941-1991, Madrid, Alianza, 1997.

ZAPATA-BARRERO, Ricard, GONZÁLEZ, Elisabet y SÁNCHEZ MONTIJANO, Elena. El discurso politico en torno a la inmigración en España y en la Unión Europea, Madrid, Ministerio de Trabajo e Inmigración. 2008.

ZAPATA-BARRERO, Ricard. "La política del discurso sobre la inmigración en España". En: ZAPATA-BARRERO, Ricard, GONZÁLEZ, Elisabet y SÁNCHEZ MONTIJANO, Elena. El discurso político en torno a la inmigración en España y en la Unión Europea, Madrid, Ministerio de Trabajo e Inmigración. 2008, pp. 117-157.

\section{DOCUMENTOS DE TRABAJO, PRENSA Y OTRAS FUENTES}

COLEGIO DE PERIODISTAS DE CATALUÑA. "Manual de estilo sobre minorías étnicas". En: Quaderns del CAC. Número 12. Enero-Abril de 2002. pp. 68-70. Disponible en: www.audiovisualcat.net [21/07/2016].

CONSEJO AUDIOVISUAL DE CATALUÑA. "Recomendaciones del Consejo del Audiovisual de Cataluña sobre el Tratamiento Informativo de la Inmigración”. En: Quaderns del CAC, Número 12. Enero-Abril de 2002, pp: 63-67. A disposición en www.audiovisualcat.net [21/07/2016].

CONSEJO DE LA UNIÓN EUROPEA. Decisión 2000/750/CE del Consejo de 27 de noviembre de 2000 por la que se establece un programa de acción 
DELICTAE, Vol. 4, No6, Jan..-Jun. $2019 \mid 35$

comunitario para luchar contra la discriminación (2001-2006). Diario Oficial de las Comunidades Europeas L 303/23, de 2 de diciembre de 2000.

COORDINADORA DE ONG DE EUSKADI DE APOYO A INMIGRANTES. Inmigración y Medios de Comunicación. Manual recopilatorio de buenas prácticas periodísticas. Consultable en: http://www.mugak.eu/gunea/obsmedios/buenpract/ [21/07/2016].

JEFATURA DEL ESTADO. Ley Orgánica 2/2009, de 11 de diciembre, de reforma de la Ley Orgánica 4/2000, de 11 de enero, sobre derechos y libertades de los extranjeros en España y su integración social. BOE $\mathrm{N}^{\circ}$ 299, de 12 de diciembre de 2009.

OBSERVATORIO EUROPEO DEL RACISMO Y LA XENOFOBIA (EUMC) y WAL, Jessica ter (Ed.). Racism and Cultural Diversity in the Mass Media. An overview of research and examples of good practice in the EU Member States 1995-2000, Viena, European Research Centre on Migration and Ethnic Relations (ERCOMER), 2002. Disponible en: http://fra.europa.eu/fraWebsite/ [27/08/2019].

PÉREZ WOLFRAM, Clara. "Las inmigrantes en la prensa: víctimas sin proyecto migratorio". En revista: $M U G A K$. No 24, Centro de Estudios y Documentación sobre Inmigración, Racismo y Xenofobia (MUGAK) de SOS Racismo, 2005. Disponible en: http://revista.mugak.eu/articulos/ [21/07/2016].

SIMÓN, Pedro. "Los inmigrantes amplian el plazo de suspensión de la huelga". El Mundo, 26 de febrero de 2000, p. 39.

UNIÓ DE PERIODISTES VALENCIANS. Manual para los periodistas sobre la protección de la infancia en los medios de comunicación. Consultable en: http://www.unioperiodistes.org_[27/08/2019]. 